\title{
Diode Pumped Solid State Laser Photoacoustic Spectrometer
}

\author{
Jeffrey S. Pilgrim and David S. Bomse \\ Southwest Sciences, Inc., 1570 Pacheco Street, Suite E-11, Santa Fe, NM 87505 \\ Phone: 505.984.1322, FAX: 505.988.9230,E-mail: jpilgrim@swsciences.com
}

\begin{abstract}
Photoacoustic spectroscopy (PAS) is well-known for trace gas detection but has been hampered by the lack of a simple tunable infrared source. DPSS lasers offer this capability and enable a PAS sensor with superior performance.

(C)2000 Optical Society of America

OCIS codes: (130.6010) Sensors; (140.3480) Lasers, diode-pumped
\end{abstract}

\section{Introduction}

Photoacoustic spectroscopy [1,2] is a well known method pioneered by Alexander Graham Bell for measuring weak optical absorbances indirectly. Optical absorption by the target compound heats the sample. The small temperature rise creates a change in pressure that is detected with a microphone. The magnitude of the pressure change depends on the product of the sample absorbance and the light source intensity. Usually, the light is chopped at an audio frequency, and the photoacoustic signal is detected using a lock-in amplifier. Photoacoustic detection is useful because modern microphones have low background noise and good linearity. However, broad implementation of PAS has been hampered by the absence of a simple tunable infrared source with moderate power.

Diode pumped solid state (DPSS) lasers span the 1-3 micron region. Individual systems can tune over 100 nanometers. In addition, these lasers readily offer powers in excess of $100 \mathrm{~mW}$. DPSS lasers are ideally suited for detecting a wide variety of trace gas species when coupled with PAS detection. Of particular importance is the ability to quantitatively detect many species of concern in the environment, in space, and in chemical and microelectronics process streams. Our present $\mathrm{Tm}^{3+}: \mathrm{YAG}$ DPSS system operates over a broad wavelength range near 2 microns sufficient to allow detection of $\mathrm{H}_{2} \mathrm{O}, \mathrm{NH}_{3}, \mathrm{CO}_{2}, \mathrm{NO}_{2}$ and $\mathrm{N}_{2} \mathrm{O}$.

\section{DPSS Laser}

The DPSS laser system of the present study is based on $\mathrm{Tm}^{3+}$ :YAG [3]. Other solid state lasers are available as shown in Fig. 1, which is not meant to be an exhaustive compilation. Additional solid state systems are continually

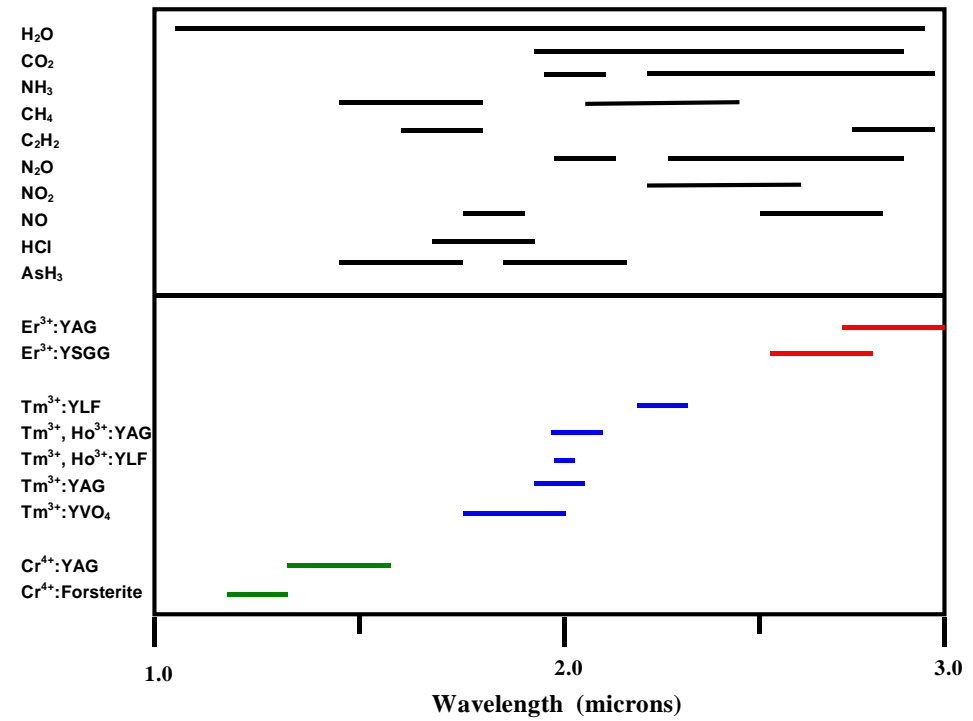

Fig. 1. Absorption regions for molecules of interest in trace gas detection are shown (upper half). A sample of the types of DPSS lasers available and their respective tuning ranges are included(lower half).

being demonstrated as well. $\mathrm{Tm}^{3+}: \mathrm{YAG}$ is perhaps the most common and extensively studied of these systems and 
provides convenient laser operation around 2 microns with a broadband tuning range from 1.9 to 2.1 microns. The free running wavelength of $\mathrm{Tm}^{3+}: \mathrm{YAG}$ is around $2015 \mathrm{~nm}$ which makes it ideal for $\mathrm{CO}_{2}$ detection. Because the crystal has a strong absorption around $780 \mathrm{~nm}$, it is ideal for pumping with commercial high powered AlGaAs diode lasers. These pump laser diodes are commonly available with $2 \mathrm{~W} c \mathrm{cw}$ output power in single stripe devices. In addition, these lasers enjoy constant improvement and some manufacturers already offer some $3 \mathrm{~W}$ cw lasers in similar packages at other wavelengths. The output power of the DPSS laser generally scales linearly with pump power above threshold. Thus, the DPSS lasers will also benefit from the continuing improvement in the pump laser diodes. In addition, there are also $\mathrm{Al}$ free diode lasers available at $780 \mathrm{~nm}$ which will lead to significantly long diode lifetimes. These types of pump lasers would be suitable for extended spaceborne applications.

\section{Photoacoustic Spectrometer}

The best photoacoustic detection limits are usually achieved in instruments utilizing resonant photoacoustic cells [4]. Resonant cells allow build up of the acoustic wave over many cycles of the resonant frequency. These resonances, which can be due to radial, azimuthal, or longitudinal modes of the cell, are typically at a few $\mathrm{kHz}$ and the amount of build up (the quality factor, or $\mathrm{Q}$, of the resonator) typically exceeds the expected loss in photoacoustic signal that would be predicted in shifting to higher frequencies.

The data of Fig. 2 were obtained using a cylindrical PAS cell. The DPSS laser was amplitude modulated at the first radial resonance of the cell. Lock-in detection at the first harmonic of the modulation frequency provides a signal proportional to the amount of absorbed power in the PAS cell. The amount of absorbed power is proportional

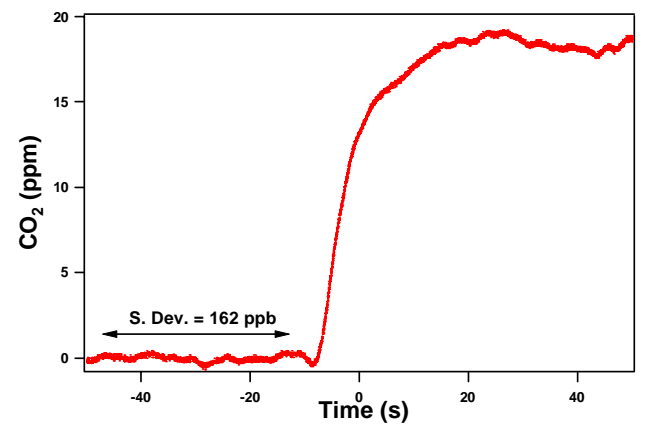

Fig.2. Photoacoustic measurement of $\mathrm{CO}_{2}$ concentration over $100 \mathrm{~s}$ during step change.

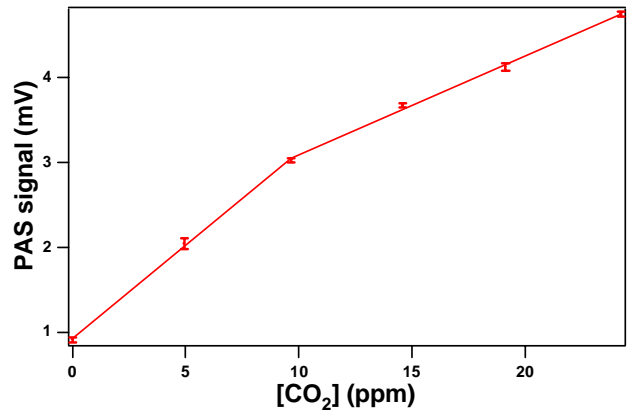

Fig.3. PAS signal calibration curve with $\mathrm{CO}_{2}$ concentration. The slope change is due to absorption saturation effects.

to the Beer's law absorbance of the sample which is proportional to $\mathrm{CO}_{2}$ concentration. The DPSS laser was utilized in free-running mode around $2015 \mathrm{~nm}$ where it is coincident with several $\mathrm{CO}_{2}$ absorption features. Even with the DPSS laser operating in broadband mode, the standard deviation of the measured signal ( $1 \mathrm{~s}$ lock-in time constant) over a $50 \mathrm{~s}$ period is equivalent to $162 \mathrm{ppb} \mathrm{CO}_{2}$. This translates into a minimum detectable absorbance of $2.4 \times 10^{-8}$ ! The data of Fig. 3 are a $\mathrm{CO}_{2}$ calibration curve obtained in a manner like that of Fig. 2 where the PAS signal is determined over $50 \mathrm{~s}$ with a $1 \mathrm{~s}$ time constant. Although small, error bars are included on each data point in Fig. 3 with the error consistently around the $150 \mathrm{ppb}$ level. The roll-over in the slope of the calibration curve is due to $\mathrm{CO}_{2}$ absorption levels extending out of the linear Beer's law regime.

\section{Future Work}

Improvements to the DPSS-based photoacoustic spectrometer will be to add broad wavelength tunability and to narrow the laser bandwidth by an order-of-magnitude while suffering only a modest reduction in output power. The resulting higher spectral density will provide significant improvement in achievable detection limits. Other efforts will be directed towards construction of DPSS lasers based on other gain media that will allow for high sensitivity trace moisture detection.

\section{References}

1. L. B. Kreuzer, J. Apply. Phys. 42, 2934 (1971).

2. M. Feher, Y. Jiang, J. P. Maier and A. Miklos, Appl. Opt. 33, 1655 (1994).

3. R. C. Stoneman and L. Esterowitz, Opt. Lett. 15, 486 (1990).

4. Optoacoustic Spectroscopy and Detection, Y-H Pao, ed., Academic Press, New York, 1977 (pp. 20-22).

This work was supported by the National Science Foundation under grant DMI-9983349. 